\title{
Usability Evaluation of a Web Academic Software Developed to Measure
}

\section{Evaluación de la usabilidad de un aplicativo web académico desarrollado a medida}

\section{Iván Menes Camejo, Gladys Lorena Aguirre Sailema, Carlos Luis Rodríguez Niama, Katherine Maribel Gallegos Carrillo, Jorge Ariel Menéndez Verdecia}

Escuela Superior Politécnica de Chimborazo, Panamericana Sur km 11/2, ECo60155 RiobambaEcuador

\section{Abstract}

University requires agility in accordance to the needs arising from technological innovation and the reform of the regulations that govern higher education, which is why the present project arises to model and implement a web application that Iván Menes Camejo imenes@espoch.edu.ec

Received: 4 December 2018 Accepted: 5 December 2018 Published: 27 December 2018

Publishing services provided by Knowledge $\mathrm{E}$

(c) Iván Menes Camejo et al. This article is distributed under the terms of the

Attribution License, which permits unrestricted use and redistribution provided that the original author and source are credited.

Selection and Peer-review under the responsibility of the SIIPRIN-CITEGC Conference Committee.
Corresponding Author: allows the entry and visualization of qualifications, and online registration under the academic regulations that govern the Technical University of Cotopaxi. For the development of this web application, Metric $V_{3}$ methodology was used, with its five stages within the life cycle of software development. Once the application was developed, it was submitted to a usability evaluation with four computer users who issued their assessments respect to ten questions related to the usability principles of Jakob Nielsen. The result was $86.5 \%$ favorable of the application for which it was necessary to previously analyze the options of "packaged" software and customized development.

\section{Resumen}

La universidad requiere de agilidad conforme a las necesidades que surgen por la innovación tecnológica y la reforma de las normativas que rigen la educación superior, por ello surge el presente proyecto para modelar e implementar una aplicación web, que permita el ingreso y visualización de calificaciones, y matriculación en línea bajo el reglamento académico que rige a la Universidad Técnica de Cotopaxi. Para el desarrollo de esta aplicación web se utilizó la metodología Métrica $V_{3}$, con sus cinco etapas dentro del ciclo de vida de desarrollo del software. Una vez el aplicativo fue desarrollado se lo sometió a una evaluación de usabilidad con cuatro usuarios informáticos que emitieron sus valoraciones con respecto a diez preguntas planteadas asociadas a los principios de usabilidad de Jakob Nielsen. El resultado arrojó un $86,5 \%$ favorable del aplicativo para el cual fue necesario analizar previamente las opciones de software "empaquetado" y desarrollo a medida.

Keywords: web application, usability, Metrica V3

\section{G OPEN ACCESS}




\section{Introducción}

La universidad requiere de agilidad y adaptación conforme a las nuevas necesidades demandadas por parte de los sectores estudiantil y docente, que surgen por la innovación tecnológica y la reforma de las normativas que rigen la educación superior en el Ecuador, pero manteniendo una integridad de la información histórica, con un cambio transparente, no solo para los usuarios; sino también para las instituciones externas que mantienen una interoperabilidad con la Universidad. Para ello es necesario actualizar las plataformas informáticas existentes y desarrollar nuevas herramientas que permitan que la Universidad se encuentre a la vanguardia, no solo en el ámbito tecnológico, sino también en cuanto a la aplicación de reglamentaciones tanto internas como de las que viene de organismos de control a nivel nacional. Como parte de esta necesidad, en el mercado se ofertan dos tipos de soluciones informáticas. Una primera que es conocida como el software 'empaquetado', cuyas principales características giran en torno al enfoque comercial de su fabricante, que busca dar el valor agregado de las mejores prácticas a su producto, resultado de su experticia en el mercado. Por otra parte, se encuentra la solución a medida, es decir un desarrollo que se centra en el cliente y en sus necesidades puntuales, y que se adapta de mejor forma a los procesos existentes. Al final, todo depende del contexto de la institución y de sus principales necesidades y los recursos que está dispuesta a emplear para alcanzar los objetivos planteados. En este sentido, se propone el desarrollo e implementación de un aplicativo web para la gestión de notas y matriculación online conforme a la reglamentación que rige a la Universidad Técnica de Cotopaxi. Para el desarrollo de esta aplicación web se utilizó la metodología Métrica $V_{3}$, que propone cinco procesos dentro del ciclo de vida de desarrollo del software: estudio de viabilidad, análisis, diseño, construcción e implantación del sistema de información. Una vez el aplicativo fue desarrollado bajo las etapas listadas anteriormente, se sometió a una evaluación de usabilidad con cuatro usuarios informáticos que emitieron sus valoraciones con respecto a diez preguntas planteadas que estuvieron asociadas a los principios de usabilidad de Jakob Nielsen. Como resultado se pudo detectar los puntos flacos de la aplicación con respecto a usabilidad, y que deberán ser revisados para mejorar la calidad del producto final. Como conclusión, se determina que una solución a medida es la mejor alternativa para el caso concreto de las necesidades de la Universidad Técnica de Cotopaxi, dadas las condiciones iniciales que implican el uso de software ya existente, así como la necesidad de que la herramienta tecnológica se adapte a las reglamentaciones vigentes. Cabe indicar que en estudio realizado por la Revista de 
la Asociación de Profesionales de Experiencia de Usuario, indica ciertas limitaciones en el proceso de evaluación heurística de Nielsen, mismo que fue aplicado en este estudio, entre lo que se destaca la falta de efectividad al momento de aplicar este tipo de evaluación en los dispositivos móviles que es a donde se orienta el desarrollo tecnológico actualmente.

\section{Contexto y estado del arte}

En particular, los mecanismos de reestructuración y reingeniería para alcanzar el diseño organizacional son los enfoques más utilizados para lograr mejoras en el funcionamiento de los sistemas de información [1]. Para lograr la mejora continua es necesario integrar la visión global estratégica de la institución con las tecnologías disponibles en el mercado. Los sistemas deben estar enmarcados en la cultura propia de cada organización [2]. Dada esta premisa, existen dos realidades a nivel del software para los procesos de las instituciones. Una primera opción que involucra el desarrollo de un software a medida, y una segunda sobre la adquisición de soluciones informáticas genéricas.

\subsection{Software a medida}

Con el software desarrollado a medida, los usuarios, los propietarios del proyecto y las partes interesadas se involucran desde el inicio y con frecuencia en el diseño y desarrollo, y puede ser planificado tomando en cuenta la integración con sistemas existentes en la empresa y permite la realización de ajustes a medida que avanza el diseño [3]. Tomando en cuenta los procesos de una organización, la solución de un software a medida puede marcar la diferencia en el cumplimiento de los objetivos trazados por parte de la dirección estratégica, puesto que se está hablando de una alta compatibilidad del software con los procesos ya establecidos en la empresa [4]. Con esta solución, si surge la necesidad de un cambio, esta solicitud se puede manejar rápidamente y la respuesta puede ser casi inmediata, debido, entre otras cosas, a la disponibilidad de todas las personas involucradas para abordar los problemas [5]. Sin embargo, cuando se trata de la implantación del software personalizado, ésta puede tomar un buen tiempo ya que el software primero debe diseñarse y desarrollarse internamente con personal, el equipo contratado sirve como personal adicional en un momento en que las cargas de trabajo son más pesadas, pero son temporales y se irán cuando ya no se necesiten [6]. 
En cuanto a los costos, el tiempo y esfuerzo necesarios para desarrollar este tipo de soluciones pueden llegar a ser costosos, puesto que el lapso para completar el producto puede ser largo y mientras los desarrolladores trabajan en el software personalizado [7].

El software personalizado se adapta, es ágil y, constituye una excelente opción para pequeñas y medianas empresas.

Como se ha planteado, uno de los objetivos que persigue la Planta de Lácteos FCPESPOCH es el generar recursos económicos autofinanciados para la ESPOCH, con la producción y comercialización de sus productos lácteos; sin embargo, se debe tomar en cuenta que dicha planta además es una unidad en la que los estudiantes universitarios realizan sus prácticas académicas. Para ser una planta rentable, combinando los objetivos de generación de recursos con los académicos, debe optimizar al máximo sus procesos de producción y comercialización.

Para lograr la optimización planteada se requeriría experimentar diferentes escenarios en los componentes que intervienen en los procesos de producción, a fin de determinar el mejor escenario posible. La experimentación in situ implicaría elevados costos para la planta, lo que no resultaría una solución factible ni deseada por el equipo directivo. Una opción económica es desarrollar un modelo de simulación computacional que permita experimentar las diferentes alternativas de funcionamiento de la planta sin intervenir en su actividad diaria, ni recurrir a costos adicionales.

\subsection{Software 'empaquetado'}

Estos paquetes están ampliamente disponibles y, a menudo, parecen costar menos que el software desarrollado a medida. A simple vista, las soluciones empaquetadas pueden ofrecer todas las características necesarias. Sin embargo, es necesario mirar un poco más profundo para conocer las características que ofrece un software "empaquetado".

El tamaño y la complejidad de las personalizaciones van desde la simple generación de informes hasta el desarrollo de complementos independientes que admiten negocios específicos. El tamaño y el tiempo de espera de dichos proyectos se pueden comparar con un proyecto de desarrollo de software de tamaño completo [8].

Los desarrolladores de software empaquetado están motivados para cumplir objetivos específicos del mercado en lugar de los de un usuario específico [9]. Con esto es posible decir que, la integración con sistemas existentes en la empresa se vuelve 
por poco probable, dadas las características de diseño que tiene el software "empaquetado", pero por otra parte trabajar con usuarios diferentes brinda a los diseñadores de software empaquetado la oportunidad de seleccionar las mejores opciones para incorporar a su solución. Comprar el software significa comprar estas mejores opciones de proceso [10]. Con respecto a la implementación del software, los desarrolladores originales rara vez participan en este proceso, no interactúan con el personal de la empresa. La desventaja es que el equipo de la compañía no estará familiarizado con el sistema, o los consultores externos no estarán familiarizados con los procesos internos de la compañía. Esto agrega tiempo considerable a los procesos de cambios, que no están garantizados a menos que lo solicitado sea considerado viable por parte de la empresa fabricante y que haya sido incluido dentro de los términos contractuales de la adquisición del software [11].

Por otra parte, la implantación del software empaquetado se puede hacer con bastante rapidez en muchos casos [12].

Se cree que el software empaquetado es más barato que el software personalizado. Sin embargo, no es posible respaldar del todo esta afirmación, especialmente cuando se toman en consideración los costos totales u ocultos. Si bien es cierto que el precio de compra de un paquete puede ser más barato que el costo de desarrollo del software personalizado, puede haber costos adicionales por encima del costo inicial que desembolsa la empresa por el producto, entre ellos, la instalación de software empaquetado puede requerir la contratación de consultores externos, o los costos asociados con los cambios requeridos en los procesos internos, las actualizaciones requeridas a lo largo de la vida del producto, la capacitación adicional del personal o el readiestramiento, la compra de nuevo hardware requerido por el sistema o incluso la adición de personal para la administración del software [13]. Comprar de fábrica proporciona a los clientes un conjunto exclusivo de funciones predefinidas, de las cuales cada cliente debe seleccionar las que sean más aplicables. Cualquier flexibilidad para estos sistemas estará representada por las opciones configurables dentro del paquete. Esta falta de flexibilidad se compensa con un mayor alcance y más funciones dentro de un sistema empaquetado [14].

\subsection{Evaluación de la usabilidad}

La usabilidad en términos generales se refiere a una característica de un producto que permita que las personas consigan sacarle el máximo partido a sus funcionalidades, posibilidades y capacidades mediante la comprensión de un uso fácil, que 
necesite poco esfuerzo y que no presente obstáculos en el proceso de aprendizaje. La definición más desarrollada de usabilidad es la de la ISO (International Organization for Standardization), que la define en su norma ISO 9241-11 de 1998: "usabilidad es el grado de eficacia, eficiencia y satisfacción con el que usuarios específicos pueden lograr objetivos específicos en contextos de uso específicos".

La evaluación de la usabilidad tiene como principales objetivos la identificación de posibles problemas de diseño, comprobar los efectos del sitio en el usuario, comprobar además la funcionalidad del producto con respecto a los objetivos establecidos y finalmente involucrar al usuario en el proceso de retroalimentación y mejora continua. Para ello se puede existen diferentes posibilidades. Una clasificación de la evaluación de la usabilidad se define por la ausencia o presencia de usuarios en el proceso, y dentro de esta taxonomía, se encuentra la evaluación heurística que es un método informal de análisis de usabilidad en el que a varios evaluadores se les presenta un diseño de interfaz y se les solicita sus comentarios basados en los principios heurísticos de Jacob Nielsen. Las ventajas de la evaluación heurística se basan en el bajo coste tanto de tiempo como de recursos, así como la facilidad de su implementación, por otra parte la principal desventaja radica en la subjetividad que implica el escoger a los evaluadores así como en la emisión de sus criterios, sin embargo estudios previos han determinado que una evaluación heurística con un conjunto de 3 a 5 evaluadores permite detectar más del 50 por ciento de los errores en la funcionalidad basados en los criterios de usabilidad del producto. Las razones antes expuestas han permitido que se escoja este método para la valoración del aplicativo web académico desarrollado a medida.

\section{Objetivos concretos y metodología de trabajo}

Los objetivos del presente trabajo se derivaron de la investigación sobre los productos académicos en el mercado frente a las necesidades de la Universidad Técnica de Cotopaxi.

\subsection{Objetivo general}

Modelar e implementar una aplicación web, que permita el ingreso y visualización de calificaciones, así como el proceso de matriculación en línea bajo el reglamento académico que rige a la Universidad Técnica de Cotopaxi. 


\subsection{Objetivos específicos}

- Indagar y analizar las ventajas y desventajas del desarrollo de software a medida.

- Indagar y analizar las ventajas y desventajas del software "empaquetado".

- Llevar a cabo un proceso de desarrollo de software para la elaboración d-una aplicación web para el ingreso de calificaciones y matriculación en línea para la Universidad Técnica de Cotopaxi.

- Medir la usabilidad de la aplicación web para el ingreso de calificaciones y matriculación en línea de la Universidad Técnica de Cotopaxi.

\subsection{Metodología del trabajo}

El aplicativo web fue desarrollado bajo la guía de la metodología Métrica Versión 3, que toma como referencia el Modelo de Ciclo de Vida de Desarrollo propuesto en la norma ISO 12.207 "Information technology-Software life cycle processes" y con ello distingue cinco procesos dentro del desarrollo de software: estudio de viabilidad, análisis del sistema de información, diseño del sistema de información, construcción del sistema de información e implantación del sistema de información. Es una metodología centrada en la clasificación y definición de los procesos del ciclo de vida del software. La metodología descompone cada uno de los procesos en actividades, y éstas a su vez en tareas. Para cada tarea se describe su contenido haciendo referencia a sus principales acciones, productos, técnicas, prácticas y participantes [15].

\section{Desarrollo Específico De La Contribución}

La Universidad Técnica de Cotopaxi, solicita una "Consultoría para el Diseño, Desarrollo e Implementación del Software Académico como parte del Sistema de Información Integrado". Dicha consultoría tiene como objeto el desarrollar los módulos del sistema académico que se detallan a continuación:

- Registro de Matriculación: módulo para la web que permitirá la gestión de las matrículas de los estudiantes en cada período académico vigente, bajo el régimen académico aprobado de la universidad. 
- Proceso de Evaluación: módulo para la web que permitirá la gestión de las diferentes calificaciones por parte del personal académico y la respectiva visualización por parte del estudiante.

\subsection{Tecnología}

La Universidad Técnica de Cotopaxi cuenta con un convenio institucional para el uso de herramientas Microsoft, debido a esto, su aplicativos propios han sido desarrollados bajo esta tecnología, y para continuar con esta línea, el aplicativo web para matriculación y gestión de calificaciones ha sido desarrollado con los siguientes recursos tecnológicos:

Recursos de Hardware

- Servidor de aplicaciones web:

- Procesador: Intel Xeon E5 2670 @ 2,60GHz

- Memoria: 4GB RAM

- Disco Duro: 84,66GB

- Servidor de base de datos:

- Procesador: Intel Xeon E5 2650 @ 2,00GHz

- Memoria: 6GB RAM

- Disco Duro: 159,66GB

Recursos Software

- Para el desarrollo:

- Sistema Operativo: Windows Server 2012 Datacenter.

- Framework: Microsoft Framework.NET 4.5.1

- Herramienta de desarrollo: Microsoft Visual Studio.Net 2013 professional.

- Motor de base de Datos: Microsoft SQL Server 2012.

- Servidor de páginas Web: Microsoft Internet Information Services 8.0 o superior.

- Navegador: Microsoft Internet Explorer V9 o superior.

- Sistema de Versionamiento: GitHub

- Para el servidor de aplicaciones: 
- Sistema Operativo: Windows Server 2012 Datacenter.

- Framework: Microsoft Framework.NET 4.5.1

- Servidor de páginas Web: Microsoft Internet Information Services 8.0 o superior.

- Navegador: Microsoft Internet Explorer V9 o superior.

- Para el servidor de base de datos:

- Sistema Operativo: Windows Server 2012 Datacenter.

- Motor de base de Datos: Microsoft SQL Server 2012.

\subsection{Requisitos}

Con el objetivo de identificar los requerimientos del aplicativo web, se realizaron reuniones con secretarias académicas de unidades académicas, idiomas y cultura física; personal técnico y con autoridades académicas de la institución. El listado de los requisitos es:

- RE-01: Los usuarios deberán autenticarse con la base de datos centralizada de la institución.

- RE-02: Realizar propuesta de matrícula conforme al cumplimiento de prerequisitos y corequisitos de la malla curricular del estudiante.

- RE-03: Verificar cruce de horarios a nivel institucional de las asignaturas seleccionadas por el estudiante de la propuesta inicial de matrícula.

- RE-04: Matricular al estudiante y emitir la orden de pago.

- RE-05: Emitir la hoja de matrícula del estudiante.

- RE-06: Registrar de las notas de evaluaciones parciales en el período académico vigente, por parte del personal docente.

- RE-07: Registrar de las notas de exámenes finales en el período académico vigente, por parte del personal docente.

- RE-08: Registrar de las notas de exámenes de gracia en el período académico vigente, por parte del personal docente.

- RE-09: Visualizar notas de las asignaturas matriculadas por parte de los estudiantes. 


\subsection{Análisis}

Con base en casos de uso se obtuvieron a groso modo las clases que intervienen en las funcionalidades requeridas para el sistema:

- Profesor: que tiene como atributos una identificación, nombres y apellidos, credenciales, unidades académicas a las que pertenece, materias y cursos que le han sido asignados. Sus acciones permitidas son ingresar calificaciones de sus materias, modificar dichas calificaciones y la visualización de las notas ingresadas, a través de interfaz web y reportes descargables. Esta clase interactúa con la clase acta de calificaciones.

- Acta de calificaciones: tiene como atributos el nombre de la asignatura, el curso, el profesor que dicta la clase, el listado de estudiantes matriculados, notas ingresadas, promedios de los estudiantes, fecha de ingreso del acta. Como acciones permitidas para esta entidad están extraer notas, guardar notas e imprimir reporte.

- Estudiante: que tiene como atributos una identificación, nombres y apellidos, credenciales, unidades académicas a las que pertenece, materias, cursos y ciclos académicos en los que se ha matriculado. Sus acciones permitidas son: acceder al sistema y visualizar su acta de calificaciones, acceder al sistema solicitar matrícula, matricularse, imprimir la orden de pago e imprimir su hoja de matrícula.

- Matrícula: tiene como atributos el estudiante asociado, las materias propuestas, los cursos de las materias propuestas, los valores de las asignaturas propuestas, el periodo académico al que pertenece, su tipo y estado. Sus acciones permitidas incluyen: carga de propuesta, verificación de cumplimiento de requisitos del estudiante asociado, cálculo de costos de asignaturas, guardar matrícula, cargar matrícula, imprimir orden de pago e imprimir hoja de matrícula.

A partir de este análisis se procedió a realizar el diseño de interfaces, de datos y clases para el aplicativo web.

\subsection{Aplicativo desarrollado}

En la Figura 1 a continuación se presenta una pantalla del resultado final del desarrollo del aplicativo web para la gestión de calificaciones y matriculación online bajo la metodología Métrica $V_{3}$. 


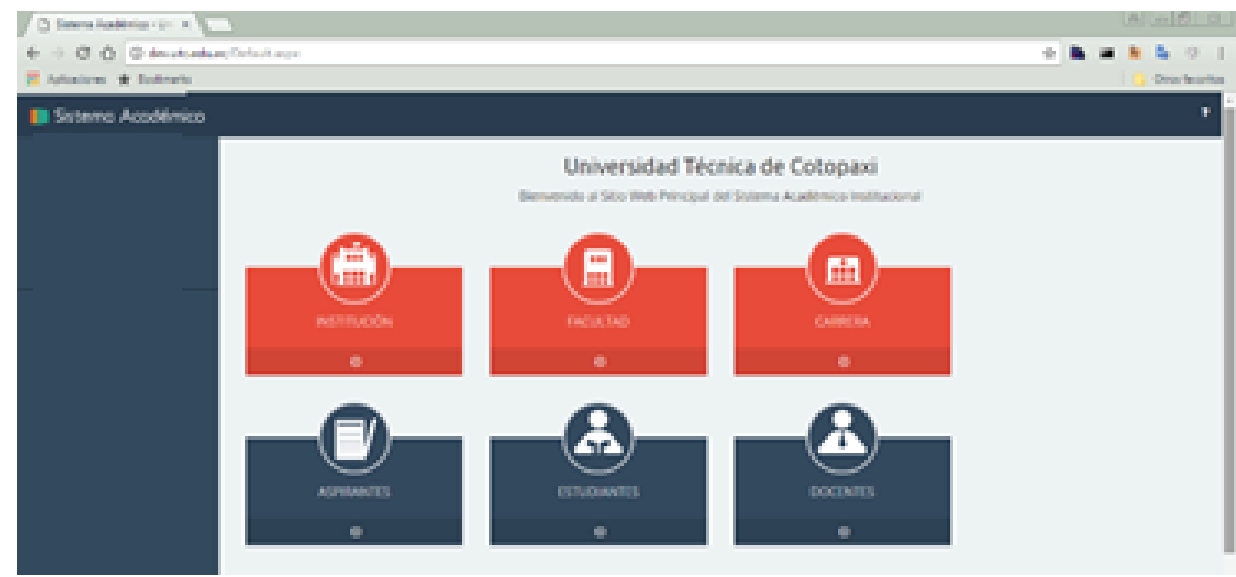

Figura 1: Captura de pantalla del aplicativo web académico desarrollado y que fue sometido a evaluación heurística con la participación de cuatro usuarios expertos.

\section{Evaluación}

La evaluación de la usabilidad del aplicativo web se lo realizó mediante el uso de la encuesta a cuatro usuarios expertos del Departamento de Tecnologías de la Información de la Universidad Técnica de Cotopaxi, quienes respondieron a un conjunto de diez preguntas que valoraban la aplicación en una escala del uno (1) al cinco (5), donde:

1. Nunca

2. Muy pocas veces

3. Algunas veces

4. Casi siempre

5. Siempre

Las preguntas realizadas a los usuarios expertos fueron:

- Pregunta 1: El diseño de la interfaz de usuario permite flexibilidad de acoplamiento en el acceso y navegación en función de diferentes estilos de aprendizaje.

- Pregunta 2: El sistema le presenta información sobre su ubicación en el directorio de navegación (migas de pan).

- Pregunta 3: Los elementos presentes se encuentran etiquetados de forma clara y precisa (Tooltips).

- Pegunta 4: La interfaz de usuario se caracteriza por la claridad, facilidad de comprensión y coherencia con los objetivos académicos del programa. 
- Pregunta 5: Los nombres presentados en los botones del software tienen relación con la acción que realiza cada uno de ellos.

- Pregunta 6: El sistema le proporciona información sobre los procedimientos a realizar en caso de un error ocurrido.

- Pregunta 7: La opción de ayuda se presenta en todas las pantallas a lo largo de la navegación por el sitio.

- Pregunta 8: El sistema le permite rectificar las elecciones tomadas.

- Pregunta 9: El sistema proporciona la información necesaria para realizar los diferentes procesos.

- Pregunta 10: El sistema previene el ingreso de datos incorrectos o no permitidos

A continuación, en la tabla 1, se resumen los resultados obtenidos en la evaluación de la usabilidad del sitio web para ingreso de calificaciones y matriculación en línea.

TABLA 1: Resultados de la evaluación de cuatro usuarios expertos sobre los diez principios de usabilidad de Jacob Nielsen sobre el aplicativo web académico.

\begin{tabular}{l|c|c|c|c|c|}
\hline Pregunta & UE1 & UE2 & UE3 & UE4 & $\sum$ \\
\hline Pregunta 1 & 5 & 4 & 4 & 5 & 18 \\
\hline Pregunta 2 & 5 & 5 & 5 & 5 & 20 \\
\hline Pregunta 3 & 4 & 3 & 4 & 4 & 15 \\
\hline Pregunta 4 & 5 & 5 & 5 & 5 & 20 \\
\hline Pregunta 5 & 4 & 4 & 4 & 5 & 17 \\
\hline Pregunta 6 & 4 & 4 & 4 & 5 & 17 \\
\hline Pregunta 7 & 2 & 3 & 2 & 2 & 9 \\
\hline Pregunta 8 & 5 & 4 & 5 & 4 & 18 \\
\hline Pregunta 9 & 4 & 5 & 5 & 5 & 19 \\
\hline Pregunta 10 & 5 & 5 & 5 & 5 & 20 \\
\hline SUMAT0RIA TOTAL & & & & $\mathbf{1 7 3}$
\end{tabular}

De los 200 puntos posibles a obtener en esta evaluación de usabilidad, se ha obtenido 173 puntos que corresponde a un $86,5 \%$ de la calificación, lo que pertenece a una valoración satisfactoria, siendo importante revisar las preguntas que han sido categorizadas como media y alta prioridad:

3. Los elementos presentes se encuentran etiquetados de forma clara y precisa (Tooltips), asociado al criterio de usabilidad "Reconocimiento antes que recuerdo".

7. La opción de ayuda se presenta en todas las pantallas a lo largo de la navegación por el sitio, asociado al criterio de usabilidad "Ayuda y documentación". 


\section{Conclusiones}

El software desarrollado a medida complementa las operaciones de la empresa, por lo que se vuelve en la solución ideal para una institución que desea mantener sus procesos intactos, y que a su vez cuenta con el personal para dedicar sus esfuerzos en el desarrollo de una solución de esta naturaleza.

El software empaquetado, ofrece a la empresa una solución que implementa las mejores prácticas del mercado, en tal virtud, la empresa que opta por esta solución, está abierta a la posibilidad de comprometer sus procesos para cambiar a otros, que el software como tal, imponga.

La evaluación con usuarios es una solución factible y efectiva para la determinación de la usabilidad de un sitio web, siempre y cuando se cuente con los usuarios expertos que emitirán sus criterios sobre el aplicativo.

A futuro se plantea aplicar la metodología de desarrollo de software Métrica V3 bajo la concepción de un marco de trabajo o tecnología ágil, es decir aplicando frameworks como Scrum o XP, con el objetivo de medir la efectividad de la aplicación ciertos principios del manifiesto ágil que incorporan mencionadas tecnologías.

\section{Referencias}

[1] J. Andrade y C. Susana, «Tecnologías de información en el diseño de la organización,» Revista Venezolana de Gerencia, vol. 6, pp. 241-256, 1998.

[2] R. Andreu, J. Ricart y J. Valor, Estrategias y sistemas de información, Madrid: McGraw-Hill, 1991.

[3] S. Sawyer y P. Guinan, «Software development: Processes and performance,» IBM Systems Journal, vol. 37, n² 2, pp. 552 - 569, 1998.

[4] J. Grudin y A. University, «Interactive systems: Bridging the gap between developers and users, 》 IEEE Computer, vol. 24, n 5, Pp. 59-69, 1991.

[5] S. Maguire, Debugging the Development Process: Practical Strategies for Staying Focused, Hitting Ship Dates, and Building Solid Teams, Redmond: Microsoft Press, 1994.

[6] R. Kling y S. lacono, "The control of information systems developments after implementation,» Communications of the ACM, vol. 27, n' 12, pp. 1218-1226, 1984

[7] B. Boehm, Software Engineering Economics, Englewood Cliffs: Prentice-Hall, 1981

[8] Y. Dittrich, S. Vaucouleur y G. Stephen, «ERP Customization as Software Engineering: Knowledge Sharing and Cooperation,» IEEE Software, vol. 26, pp. 41-47, 2009. 
[9] R. Andersson y A. Nilsson, «The Standard Application Package Market: An Industry in Transition.,» de Advancing Your Business: People and Information Systems in Concert, EFI yearbook., Stockholm, Stockholm School of Economics, 1996, p. Chapter III.

[10] A. Kjær y K. Madsen, «Customer-vendor co-operation,» Information Technology \& People, vol. 10, n³, pp. 205-223, 1997.

[11] R. Klepper y $\mathrm{H}$. Curt, "Trends in use and management of application package software,» Information Resources Management Journal, vol. 5, n 4, pp. 33-37, 1992.

[12] M. Krishnan, «The role of team-factors in packaged software quality,» Information Technology \& People, vol. 11, n 1, pp. 20-36, 1998.

[13] B. Light, "The maintenance implications of the customization of ERP software,» Software: Evolution and Process, vol. 13, n 6, p. 415-429, 2001.

[14] E. Carmel y S. Becker, «A process model for packaged software development,» IEEE Transactions on Engineering Management, vol. 41, n 5, Pp. 50-61, 1995.

[15] Portal de Administración Electrónica, «Métrica v.3,» Ministerio de Hacienda y Función Pública, [En línea]. Available: https://administracionelectronica.gob. es/pae_Home/pae_Documentacion/pae_Metodolog/pae_Metrica_vz.html\#. WoL86ejOXIU. [último acceso: 0106 2017]. 